\title{
Externe Demokratieförderung der Europäischen Union - die Instrumentenwahl der EU aus vergleichender Perspektive
}

\author{
Annette Jünemann und Michèle Knodt*
}

Das Thema der externen Demokratieförderung durch die Europäische Union steht zur Zeit ganz oben auf der politischen Agenda. Indem die Wertegemeinschaft Europäische Union ihr Modell legitimen demokratischen Regierens in Drittstaaten exportiert, hat sie Begriffe wie Demokratie, Menschenrechte, Rechtsstaatlichkeit und ,good governance zu Referenzgrößen ihres auswärtigen Regierens gemacht. Entsprechend dieser Entwicklung hat sich nun auch die Forschung dem Thema der externen Demokratieförderung zugewandt. Der überwiegende Teil dieser Forschungsbemühungen verbleibt jedoch in Einzelfallstudien zu unterschiedlichen Drittstaaten ${ }^{1}$ respektive Drittstaaten innerhalb einer Region. ${ }^{2}$ Systematisch vergleichende Studien finden sich im Vergleich unterschiedlicher externer Demokratisierer ${ }^{3}$ oder im Bereich der EU-Beitrittskandidaten. ${ }^{4}$ In diesem Beitrag wird die EU-Demokratieförderung in Nichtkandidatenstaaten einer vergleichenden Analyse unterzogen.

Die bisher in der Literatur vorherrschende Erklärung zur Strategie der externen Demokratieförderung geht davon aus, dass die Europäische Union im Sinne einer ,Innen-Außenanalogie' ihre internationale Umwelt nach denselben Werten und Prinzipien gestalten will, die im Inneren der Gemeinschaft gelten. ${ }^{5}$ Um dieses Ziel umzusetzen, machte sie die Demokratieförderung zur Querschnittsaufgabe aller Bereiche ihrer Außenbeziehungen: Handelspolitik, Entwicklungszusammenarbeit, auswärtige Kulturpolitik sowie Außen-, Sicherheitsund Verteidigungspolitik. Insofern kann man von einem , democracy mainstreaming 'sprechen, das sich unter anderem in sogenannten Demokratieklauseln manifestiert, die die Europäische Union seit 1995 in alle ihre Abkommen mit Drittstaaten einfügt. ,Democracy mainstreaming ' bedeutet jedoch nicht, dass die klassischen Instrumente der Demokratieförderung - politische Konditionalität, politischer Dialog sowie finanzielle Anreize und ,capacity building - von der Europäischen Union in allen Ländern in gleicher Weise eingesetzt würden. Vielmehr zeigt die eingehende Analyse vor allem der neueren Abkommen, dass sich die Fassungen der Demokratieklauseln bezüglich ihrer Schärfe und Verbindlichkeit erheblich voneinander unterscheiden. Zudem variiert die Demokratisierungspolitik der Europäischen

* Prof. Dr. Annette Jünemann, Institut für Internationale Politik, Helmut-Schmidt-Universität, Universität der Bundeswehr Hamburg.

Prof. Dr. Michèle Knodt, Institut für Politikwissenschaft, Technische Universität Darmstadt.

1 Vgl. u.a. Susanne Gratius: Kuba unter Castro - Das Dilemma der dreifachen Blockade. Die kontraproduktive Politik der „Demokratieförderung“ seitens der USA und der EU, Opladen 2003; Siegmar Schmidt: Die Demokratie- und Menschenrechtsförderung der Europäischen Union unter besonderer Berücksichtigung Afrikas. Arbeitspapiere zu Problemen der Internationalen Politik und der Entwicklungsländerforschung Nr. 28, München 1999.

2 Vgl. u.a. Richard Gillespie/Richard Youngs (Hrsg.): The European Union and Democracy Promotion: The Case of North Africa, London 2002.

3 Vgl. das laufende Projekt von Peter Schlotter an der Hessischen Stiftung Friedens- und Konfliktforschung (HSFK) ,Demokratisierung von Außen? Internationale Organisationen im Südkaukasus seit den 1990er Jahren“.

4 Vgl. Frank Schimmelfennig/Ulrich Sedelmeier (Hrsg.): The Europeanization of Central and Eastern Europe, Ithaca 2005.

5 Vgl. Dirk Peters/Wolfgang Wagner: Die Europäische Union in den internationalen Beziehungen, in: Katharina Holzinger u.a. (Hrsg.): Die Europäische Union. Theorien und Analysekonzepte, Wiesbaden 2005, S. 215-272, hier S. 215-216. 
Union, über die Formulierung der Demokratisierungsklausel hinaus, hinsichtlich der Instrumenten- und Strategiewahl sowie deren Implementierung.

Wie lassen sich diese Unterschiede erklären? Warum entscheidet sich die Europäische Union in einigen Fällen für eher negative Instrumente, wie etwa Sanktionen, Isolation, scharfe Konditionalitätsklauseln oder die Stützung oppositioneller Zivilgesellschaften, während sie in anderen Fällen lediglich demokratische Standards anmahnt und sich mit der Pflege eines politischen Dialogs begnügt? Bisher sind in der Literatur kaum systematisierende Antworten auf diese Fragen gegeben worden.

Im Fokus dieses Beitrags steht deshalb die Frage, nach welchen Kriterien die Europäische Union Strategien und Instrumente zur Förderung der Demokratie in unterschiedlichen Ländern auswählt und in wie weit sie die einmal gewählten Instrumente auch praktisch umsetzt. Dabei soll die Demokratisierungspolitik noch nicht bewertet werden, weder normativ noch hinsichtlich ihrer Effizienz und Effektivität. Das Erkenntnisinteresse richtet sich ganz bewusst auf die eingegrenzte Frage, wie die Europäische Union ihre Demokratieförderung gestaltet und von welchen Kriterien sie sich dabei leiten lässt. Die empirische Basis dieses Beitrags bildet neben eigenen Recherchen sowohl eine Konferenz der Autorinnen zu diesem Thema im November 2005 als auch der daraus entstandene Tagungsband. ${ }^{6}$ Der Beitrag skizziert zunächst die Bedingungen der Instrumenten- und Strategiewahl der EU-Demokratisierungspolitik. Dem folgt eine Zusammenschau der in diesem Zusammenhang wichtigsten empirischen Ergebnisse unserer Analyse. Ein kurzes Schlusskapitel wird vor allem Anknüpfungspunkte für weiterführende Forschungsarbeiten aufzeigen.

\section{Bedingungen der Instrumenten- und Strategiewahl der EU}

Stellt man sich die Frage nach den Bedingungen, unter denen die Europäische Union ihre Instrumente für die Demokratieförderung in Drittstaaten auswählt, so kann man grundlegend drei Dimensionen unterscheiden: das europäische Mehrebenensystem, die ,Resonanzstruktur' des Drittstaats beziehungsweise der dort relevanten Eliten und die Interdependenzen zwischen der Europäischen Union und dem Drittstaat. Hinzu kommt als Kontextvariable das internationale Umfeld, das ebenfalls einen Einfluss auf die europäische Demokratisierungspolitik ausüben kann.

\section{Das europäische Mehrebenensystem}

Da die Demokratieförderung unter anderem durch Außenhandelsabkommen der Europäischen Union und somit im Rahmen von gemischten bilateralen Verträgen durchgeführt wird, befinden wir uns im Bereich der geteilten Kompetenzen zwischen der Union und ihren Mitgliedstaaten. Der Bereich der geteilten Kompetenzen weist den Mitgliedstaaten eine wichtige Rolle zu, denn an den entscheidenden Stellen des Entscheidungsprozesses - wenn es etwa um die Verhängung von Sanktionen geht - beschließt der Rat einstimmig. Durch die Möglichkeit von Vetopositionen ist die Europäische Union bei der Ausgestaltung ihrer externen Demokratisierungspolitik also in einem erheblichen Ausmaß abhängig von den Interessen ihrer Mitgliedstaaten. Ihr Mehrebenencharakter wird immer dann entscheidend, wenn einzelne Mitgliedstaaten besondere Beziehungen zu einem Drittstaat unterhalten, von denen

6 Vgl. den Tagungsbericht von Sonja Grimm: Demokratieförderung durch die Europäische Union zwischen Anspruch und Wirklichkeit, in: integration 2/2006. Ein Sammelband erscheint in Kürze im Nomos Verlag: Annette Jünemann/Michèle Knodt (Hrsg.): The EU as an External Democracy Promoter. Die externe Demokratieförderung der EU. Schriftenreihe des Arbeitskreises Europäische Integration, Baden-Baden (im Erscheinen). 
sie nationale Interessen ableiten, die mit dem EU-Interesse an einer entweder besonders harten oder einer besonders weichen Demokratisierungspolitik kollidieren.

Gleichzeitig gilt, dass im Politikfeld der Demokratieförderung, gerade weil sie sich im Bereich der geteilten Kompetenzen abspielt, die Gemeinschaftsebene von größerer Bedeutung ist als sonst in der Außenpolitik üblich. Dies gilt vor allem für die Europäische Kommission, weniger für das Europäische Parlament. Bei der Analyse des Einflusses der Gemeinschaftsebene auf die EU-Demokratisierungspolitik ist des Weiteren die sogenannte ,Brüsselisierung' zu berücksichtigen. Damit werden (nicht intentionale) Angleichungsprozesse der Perzeptionen, Problemdefinitionen und Problemlösungsstrategien aufgrund langjähriger gemeinsamer Zusammenarbeit mit immer den gleichen in Brüssel tätigen Akteuren bezeichnet; Akteure die auf unterschiedliche Weise und auf unterschiedlichen Ebenen in die Entscheidungsprozesse der Europäischen Union involviert sind.

\section{Resonanz innerhalb des Drittstaats}

Ein entscheidender Faktor für die Strategie und Instrumentenwahl der EU-Demokratisierungspolitik ist der politische Zustand des Drittstaates und dabei insbesondere die dort vorzufindende , Resonanzstruktur'. Handelt es sich beispielsweise um einen fragilen, einen zerfallenden oder einen gescheiterten Staat? Davon hängt ab, inwieweit Sicherheitsrisiken von diesem Staat ausgehen, die bei der Strategiewahl zu berücksichtigen sind. Wie pluralistisch ist die Gesellschaft? Finden sich in der politischen Klasse und/oder der Zivilgesellschaft mehr oder weniger kooperative Ansprechpartner für eine demokratiepolitische Zusammenarbeit mit der Europäischen Union? Je nachdem, wird die Europäische Union unterschiedliche Kooperationsanreize zum Einsatz bringen müssen. Besonders schwierig dürfte sich die externe Demokratieförderung in Ländern gestalten, in denen extrem autoritäre Systeme oder eine fehlende beziehungsweise schwache Staatlichkeit keine Resonanzstrukturen entstehen lassen, an die mit dem zur Verfügung stehenden Instrumentarium angeknüpft werden könnte.

\section{Interdependenzen zwischen der Europäischen Union und einem Drittstaat}

Relevant für die Strategie- und Instrumentenwahl der EU-Demokratisierungspolitik sind des Weiteren wirtschaftliche und politische Interdependenzen zwischen der Europäischen Union und einem Drittstaat. Negative Instrumente können nur in der Beziehung mit solchen Ländern erfolgreich zum Einsatz gebracht werden, die wirtschaftlich und/oder politisch asymmetrische Interdependenzen zugunsten der Europäischen Union aufweisen. In solchen asymmetrischen Interdependenzbeziehungen können sich den (schwächeren) Drittstaaten jedoch Kooperationsopportunitäten durch andere Demokratisierer bieten (beispielsweise die USA, regionale Hegemonialmächte oder Internationale Organisationen), die Symmetrisierungseffekte auslösen, die der Europäischen Union ebenfalls die Anwendung negativer Instrumente erschweren.

\section{Internationaler Kontext}

Als Kontextvariable soll nicht zuletzt die internationale Umwelt beziehungsweise das veränderte Verhalten anderer externer Demokratieförderer konzeptionalisiert werden. Darunter fallen sowohl Veränderungen der internationalen Beziehungen systemischer Art - wie etwa die veränderte Sicherheitslage nach dem 11. September 2001 - als auch Strategieänderungen anderer externer Demokratisierer wie der USA oder der Vereinten Nationen. Letzteres beinhaltet sowohl das Konkurrenzverhältnis der Europäischen Union zu anderen Staaten als auch ihre rechtliche Einbindung in internationale Organisationen sowie eventuelle arbeitsteilige Strategien. 


\section{Empirische Erkenntnisse über die Instrumenten- und Strategiewahl der EU-Demo- kratieförderung}

\section{Implikationen des auswärtigen Regierens im Mehrebenensystem}

Wie oben bereits festgestellt, eröffnet die Demokratieförderung durch ihr im Bereich der geteilten Kompetenzen angesiedeltes Instrumentarium Vetopositionen für die Mitgliedstaaten. Daher kommt den bilateralen Beziehungen ,interessierter Mitgliedstaaten ' zu einem Drittstaat besondere Bedeutung zu. Es hat sich gezeigt, dass solch enge bilaterale Beziehungen den Handlungsspielraum der Europäischen Union erheblich einengen können und zwar meistens dahingehend, dass sie sich gezwungen sieht, auf den Einsatz negativer Instrumente der Demokratieförderung zu verzichten. Laura Feliu hat diesen Zusammenhang unter dem Begriff des ,Paradoxes der Außenpolitik' folgendermaßen ausdifferenziert: Je enger die Beziehungen zwischen einem EU-Mitgliedstaat und einem Drittstaat sind, um so mehr Möglichkeiten der Einflussnahme ergeben sich für die Gemeinschaft. Diese Möglichkeiten bleiben jedoch ungenutzt, weil mit der Dichte der Beziehungen auch die Dichte nationaler Interessen zunimmt, die Zurückhaltung in der Anwendung vor allem negativer Instrumente der Demokratieförderung opportun werden lassen. Im Ergebnis wird somit die Förderung der Demokratie durch nationale - sicherheitspolitische und/oder wirtschaftliche - Erwägungen neutralisiert. ${ }^{7}$

Empirische Bestätigung findet das außenpolitische Paradox im Maghreb, wo sich die direkt betroffenen Nachbarn Spanien, Italien und Frankreich aus vorwiegend stabilitätspolitischen Gründen für eine äußerst zurückhaltende EU-Demokratieförderung stark machen und bislang jeglichen Einsatz negativer Instrumente verhindern konnten. ${ }^{8}$ Analoges galt für die Politik der damaligen EG gegenüber dem Apartheidregime Südafrikas. Als ehemalige Kolonialmacht war es vor allem Großbritannien, das aus handelspolitischen Gründen und mit Rücksicht auf die britischen Bevölkerungsanteile innerhalb der weißen Minderheit Südafrikas eine harte Antiapartheidpolitik der Gemeinschaft verhinderte. ${ }^{9}$ In der Empirie finden sich umgekehrt aber auch Beispiele dafür, dass besondere bilaterale Beziehungen zwischen einem Drittstaat und einem EU-Mitgliedstaat positiv wirken können. Dies ist überall da der Fall, wo zwar enge, oft historisch gewachsene bilaterale Beziehungen bestehen, die jeweiligen EU-Mitgliedstaaten aber nicht befürchten müssen, dass sich eine konsequente EU-Demokratieförderung negativ auf die eigene Sicherheit oder Wirtschaft auswirkt. Als Beispiel sei hier Ghana genannt, wo sich innerhalb der Europäischen Union vor allem Dänemark für einen konsequenten Einsatz des demokratiepolitischen Instrumentariums einsetzt. ${ }^{10}$ Wieder anders gestaltet sich die Situation auf dem Balkan. Hier nehmen auch die Mitgliedstaaten, die über besondere bilaterale Beziehungen zu einzelnen Ländern der Region verfügen, etwaige negative Auswirkungen der EU-Demokratisierungspolitik in Kauf, weil das Interesse an einer schnellen Demokratisierung dieser potenziellen Beitrittskandidaten bei allen Mitglied-

7 Vgl. Laura Feliu: A Two-Level-Game: Spain and the Promotion of Democracy and Human Rights in Morocco, in: Annette Jünemann (Hrsg.): Euro-Mediterranean Relations after September 11: International, Regional and Domestic Dynamics, London 2004, S. 90-111, hier S. 92.

8 Vgl. Annette Jünemann: Support for Democracy or Fear of Islamism? Europe and Algeria, in: Kai Hafez (Hrsg.): The Islamic World and the West: An Introduction to Political Cultures and International Relations, London 2000, S. 103-126.

9 Vgl. Siegmar Schmidt: The EU Democracy Assistance in Africa: The cases of South Africa and the Democratic Republic of Congo (DRC), in: Annette Jünemann/Michèle Knodt (Hrsg.): The EU as an External Democracy Promoter.

10 Vgl. Gordon Crawford: Assessing EU Democracy Promotion in Africa: The case of Ghana, in: Annette Jünemann/ Michèle Knodt (Hrsg.): The EU as an External Democracy Promoter. 
staaten nationale Partikularinteressen überwiegt. ${ }^{11}$ Letztendlich scheint es auch ,interessierte Mitgliedstaaten' zu geben, deren Interesse eher in der symbolischen Politik liegt. Hier ist etwa an das zunehmende Engagement der baltischen Staaten in Zentralasien zu denken, das weniger durch wirtschaftliche- oder Sicherheitsinteressen erklärt werden kann, als vielmehr durch das Bedürfnis, nach eigener erfolgreicher Demokratisierung nunmehr selbst als externer Demokratisierer tätig zu werden.

Nachdem nachgewiesen ist, welch besondere Rolle die bilateralen Beziehungen einzelner Mitgliedstaaten für die Strategiewahl der EU-Demokratisierungspolitik spielen, gewinnt auch der Faktor eines Regierungswechsels in einem derart interessierten Mitgliedstaat an Relevanz. Deutlich erkennbar wird dies etwa an der Einflussnahme Spaniens auf die Demokratieförderung der Europäischen Union in einzelnen lateinamerikanischen und karibischen Drittstaaten, die sich durch den Wechsel zwischen der Regierung Aznar und Zapatero entscheidend veränderte: weg von einer Politik, die die Europäische Union im Sinne des außenpolitischen Paradoxes behinderte und hin zu einer aktiven Bestärkung der EU-Demokratieförderung.

Aber auch die Gemeinschaftsebene gewinnt für die Gestaltung der externen Demokratieförderung zunehmend an Bedeutung. Hier ist vor allem an die Europäische Kommission zu denken, die mit der Administration der unterschiedlichen Projekte und Programme betraut ist. Dabei hat sie politische Lernprozesse durchlaufen, die sich weniger auf die Strategie- und Instrumentenwahl als vielmehr auf deren Implementierung auswirken. Im Kontext der Euro-Mediterranen Partnerschaft, um ein Beispiel zu geben, lässt sich der Lernprozess der Kommission an ihrem Umgang mit der Zivilgesellschaft ablesen. Zu Beginn förderte sie vor allem westlich orientierte und säkularisierte Vertreter der Zivilgesellschaft, die sie nicht aufgrund von politischen oder theoretischen Kriterien ausgewählte, sondern die schlicht von anderen europäischen Projektpartnern empfohlen wurden. ${ }^{12}$ Zehn Jahre später kann man beobachten, dass das Management zivilgesellschaftlicher Projekte professionalisiert wurde. Die Auswahl der Projektpartner erfolgt nun unter systematischerer Berücksichtigung der spezifischen Struktur der Zivilgesellschaft in der Mittelmeerregion, was eine vorsichtige Einbeziehung des moderaten Spektrums islamischer Gruppierungen impliziert. Dabei spielt, ähnlich wie auf Ebene der Mitgliedstaaten, der Wechsel an der Spitze der Kommission eine nicht zu unterschätzende Rolle. Während der Amtszeit von Kommissionspräsident Romano Prodi und dem Kommissar für Außenbeziehungen, Chris Patten, wurde der Zusammenarbeit mit der Zivilgesellschaft spürbare Bedeutung zugemessen, wie innerhalb des Europäischen Parlamentes und einschlägiger NGOs positiv vermerkt wurde. Seit der Amtsübernahme durch José Manuel Barroso und Benita Ferrero-Waldner hat das politische Interesse jedoch spürbar nachgelassen. ${ }^{13}$

Ebenfalls dem Bedeutungszuwachs der Gemeinschaftsebene zuzurechnen ist die Ansiedlung der meisten für die Gemeinsame Außen- Sicherheits- und Verteidigungspolitik (GASP und ESVP) relevanten EU-Institutionen direkt in Brüssel. Diese Brüsselisierung bewirkt horizontale und vertikale Anpassungs- und Angleichungsprozesse bei den Akteuren der einzelnen Mitgliedstaaten auf allen Ebenen des EU-Systems. Sie trägt damit indirekt dazu bei, dass diese weitgehend intergouvernementalen Politikfelder unterhalb der Schwelle der zur Zeit nicht zu realisierenden Integration kohärenter werden. Damit stärkt die Brüsselisierung

11 Vgl. Susan Stewart: EU Democracy Promotion in the Western Balkans, in: Annette Jünemann/ Michèle Knodt (Hrsg.): The EU as an External Democracy Promoter.

12 Vgl. Annette Jünemann: From the bottom to the top: Zivilgesellschaft und transnationale NGOs in der EuroMediterranen Partnerschaft, in: Michèle Knodt/Barbara Finke (Hrsg.): Europäische Zivilgesellschaft. Konzepte, Akteure, Strategien, Wiesbaden 2005, S. 249-270.

13 Vgl. Interviews von Annette Jünemann mit Mitgliedern des Europäischen Parlaments, Vertretern des EuroMediterranean-Human-Rights-Network und der Friedrich-Ebert-Stiftung, April 2006 in Brüssel. 
nicht zuletzt das außenpolitische Profil der Gemeinschaft im internationalen System. Das Bemühen um ein kohärenteres Auftreten nach außen könnte, zumindest theoretisch, den oben beschriebenen oft negativen Einfluss einzelner Mitgliedstaaten auf die EU-Demokratisierungspolitik relativieren. Für diese These ließen sich allerdings (noch) keine empirischen Belege finden. Vielmehr hat sich gezeigt, dass die Strategie- und Instrumentenwahl der externen Demokratieförderung aufgrund der in Brüssel gesetzten außenpolitischen Prioritäten nicht allein auf Veränderungen in den jeweiligen Drittstaaten zielt, sondern zu einem erheblichen Teil auf eine Festigung und Stärkung der GASP. Vor diesem Hintergrund gewinnt die These von Stefan Brüne an Plausibilität, der die neue Afrikastrategie der EU in erster Linie als Instrument europäischer Selbstverständigung interpretiert und erst in zweiter Linie als operative Strategie zur Erreichung der ins Auge gefassten demokratiepolitischen Ziele. ${ }^{14}$

\section{Implikationen symmetrischer und asymmetrischer Interdependenzen}

Die Instrumenten- und Strategiewahl hängt zu einem großen Teil von den Interdependenzen zwischen der Europäischen Union und einem Drittstaat ab. Je größer das wirtschaftliche und politische Potenzial des Drittstaates in seiner Beziehung zur Europäischen Union ist und je mehr alternative Kooperationsmöglichkeiten für diesen Staat bestehen, desto zurückhaltender ist die Union - zwangsläufig - im Gebrauch negativer Instrumente. Das prägnanteste Beispiel für diesen Zusammenhang ist die Kooperation mit China. Sie beruht auf einem handels- und wirtschaftspolitischen Abkommen von 1985, das 1994 und 2002 durch einen politischen Dialog zwischen der Europäischen Union und China ergänzt wurde. Negative Instrumente wie die politische Konditionalität haben keinen Eingang in das Abkommen gefunden. Angesichts der fehlenden Möglichkeiten einer direkten demokratiepolitischen Einflussnahme versucht die Union im Bereich des Handels zumindest Werte wie nachhaltige Entwicklung und Rechtsstaatlichkeit über den Umweg der Welthandelsorganisation (WTO) durchzusetzen. Seit Beginn des (mittlerweile abgeschlossenen) Beitrittsprozesses Chinas zur WTO hat die Europäische Union ihre bilateralen Verhandlungen mit China zunächst mit der WTO-Arena gekoppelt, um die Verhandlungen dann gänzlich auf das multilaterale Gremium zu verlagern. Damit verschaffte sich die Gemeinschaft Zugang zu möglichen Bündnispartnern und entwickelte gleichzeitig eine Legitimationsstrategie zur besseren Durchsetzung von multilateral anerkannten Prinzipien und Normen innerhalb der WTO-Arena. ${ }^{15}$ Diese Legitimationsstrategie verweist auf das Phänomen der immer stärkeren Vernetzung internationaler Organisationen, das mit einem zunehmenden Ex- und Import von Prinzipien und Normen einhergeht. ${ }^{16}$ Sie lässt sich auch in der Verankerung der externen Demokratieförderung im neuen EU-Verfassungsvertrag erkennen. So koppelt die Gemeinschaft in Artikel I-3,4 ihre Werte direkt an jene Werte, die schon in der Charta der UN verankert sind. Die Werte hingegen, die sich in allen Abkommen mit Drittstaaten innerhalb der Demokratieklauseln finden, werden in einen internationalen Kontext gestellt, der je nach Abkommen variiert. So bildet der internationale Referenzrahmen im Fall der Abkommen im AKP-Bereich die Universale

14 Vgl. Stefan Brüne: Testfall Äthiopien: Die neue Afrikastrategie der Europäischen Union; Siegmar Schmidt: EU Democracy Assistance in Africa, in: Annette Jünemann/Michèle Knodt (Hrsg.): The EU as an External Democracy Promoter.

15 Vgl. Michèle Knodt: Regieren im erweiterten europäischen Mehrebenensystem: Internationale Einbettung der EU in die WTO, Baden-Baden 2005.

16 Vgl. Michèle Knodt/Markus Jachtenfuchs: Regieren in internationalen Institutionen, in: Michèle Knodt/Markus Jachtenfuchs (Hrsg.): Regieren in internationalen Institutionen, Opladen 2002, S. 9-28; Sebastian Oberthür/Thomas Gehring (Hrsg.): Institutional Interaction in Global Environmental Governance: Synergy and Conflict Among International and EU Policies, Cambridge 2006. 
Erklärung der Menschenrechte und damit die UN, während in den Stabilitäts- und Assoziationsabkommen mit den Balkan-Ländern eindeutig auf die OSZE verwiesen wird. ${ }^{17}$

Empirische Belege gibt es auch für den eingangs konstruierten Fall, dass ein ökonomisch und politisch schwaches Land außenpolitische Opportunitäten erhält, die seine Abhängigkeit von der Europäischen Union relativieren. So ist etwa im Fall Kuba zu beobachten, dass die neuen Opportunitäten durch den Regierungswechsel in Venezuela zu Hugo Chavéz und dessen großzügige Energiehilfe an Kuba dazu geführt haben, dass Kuba es sich leisten kann, seine Beziehungen zur Europäischen Union stark zurückzufahren. Damit hat sich der ohnehin schon geringe Einfluss der Gemeinschaft aus das Regime Fidel Castros weiter verringert. Anders als im Fall Kuba, dessen Resonanzstruktur von autoritärer Staatlichkeit gekennzeichnet ist, verhält es sich im Fall von Afghanistan, einem Land, dem es an funktionierenden staatlichen Strukturen mangelt. Dieses ökonomisch und politisch schwache Land verfügt aufgrund zahlreicher Geldgeber über jede Menge Opportunitäten, mit denen es sich der demokratiepolitischen Einflussnahme der Europäischen Union entziehen könnte. Dennoch treten die zu erwartenden Verhaltensweisen hier nicht ein. Die extrem schwache afghanische Regierung ist gar nicht in der Lage, ihre verschiedenen Geldgeber gegeneinander auszuspielen. Die Union verzichtet vielmehr von sich aus auf den Einsatz negativer Instrumente, da Sanktionen die humanitäre Not verschlimmern und damit auch die Destabilisierung des Landes vorantreiben würden. Mehr als positive Anreize stehen der Gemeinschaft angesichts der schwierigen Resonanzstrukturen in Afghanistan nicht zur Verfügung. ${ }^{18}$

Interdependenzen können für die Strategie- und Instrumentenwahl der EU-Demokratieförderung auch dann eine Rolle spielen, wenn sie gar nicht zwischen der Gemeinschaft und dem spezifischen Drittstaat bestehen, sondern zwischen der Gemeinschaft und einer regionalen Hegemonialmacht. So hält sich die Europäische Union beispielsweise in den fünf zentralasiatischen Republiken Kasachstan, Kirgistan, Tadschikistan, Turkmenistan und Usbekistan aus Rücksicht auf Russland demokratiepolitisch extrem zurück. Mit der ehemaligen Supermacht Russland verbinden die Gemeinschaft äußerst asymmetrische sicherheits- und energiepolitische Interdependenzen, die für diese Zurückhaltung ausschlaggebend sind. ${ }^{19}$

Eine freiwillige Beschränkung der Europäischen Union auf positive Instrumente findet sich nicht zuletzt in ihrer Demokratisierungspolitik im südlichen Mittelmeerraum. Starke wirtschaftliche Asymmetrien würden hier zwar den Einsatz negativer Instrumente möglich machen. Abgesehen von der oben bereits erwähnten Vetoposition Frankreichs, Spaniens und Italiens steht jedoch eine weitere Erwägung einer härteren Gangart entgegen. Seit dem 11. September 2001 haben sich viele der autoritären arabischen Regime als Partner im Kampf gegen den internationalen Terrorismus profiliert und damit Interdependenzen im sicherheitspolitischen Bereich geschaffen, deren Asymmetrie nunmehr zu Lasten der EU geht. ${ }^{20}$

\section{Demokratie als Garant von Sicherheit und Stabilität in der Nachbarschaft}

Gemäß der Theorie des demokratischen Friedens ist der Wert der Demokratie nicht allein normativ zu bemessen; sie gilt auch als Garant von Stabilität und Sicherheit in den internati-

17 Vgl. Marise Cremona: Values in the EU constitution: The External Dimension, in: Susan Millns/Miriam Aziz (Hrsg.): Values in the Constitution of Europe, London (im Erscheinen).

18 Vgl. Florian Kühn: Das Engagement der Europäischen Union zur Demokratisierung Afghanistans, in: Annette Jünemann/Michèle Knodt (Hrsg.): The EU as an External Democracy Promoter.

19 Vgl. Alexander Warkotsch: Die Demokratisierungspolitik der EU in Zentralasien, in: Annette Jünemann/Michèle Knodt (Hrsg.): The EU as an External Democracy Promoter.

20 Vgl. Annette Jünemann: Security Building in the Mediterranean after September 11, in: Annette Jünemann (Hrsg.): Euro-Mediterranean Relations, S. 1-20. Auch erschienen in: Mediterranean Politics 2-3/2003, Special Issue, S. 1-20. 
onalen Beziehungen. Von Demokratien wird erwartet, dass sie nicht nur ihre internen, sondern auch ihre externen Konflikte mit friedlichen Mitteln lösen, zumindest wenn es sich um Konflikte zwischen Demokratien handelt. ${ }^{21}$ Aus dieser Perspektive ist die externe Demokratieförderung der Europäischen Union auch als Stabilitäts- und Sicherheitspolitik zu verstehen; als Versuch, den Import von Instabilität und Unsicherheit aus Osteuropa und dem südlichen Mittelmeerraum zu vermeiden. ${ }^{22}$ Dies erklärt den empirischen Befund, dass die EUDemokratieförderung in der unmittelbaren Nachbarschaft, und nur dort, den umfassenden Einsatz des gesamten demokratiepolitischen Instrumentariums vorsieht. Institutioneller Rahmen dafür ist die Europäische Nachbarschaftspolitik. Hier wird unter dem Stichwort des ,joint ownership “ versucht, mit den Drittstaaten partnerschaftliche Vereinbarungen zu schließen, die auf gemeinsamen Werten aufbauen. Statt alle Nachbarstaaten in einen von der Europäischen Union vorgegebenen Rahmen zu pressen, werden in bilateralen Aktionsplänen auf die Situation des jeweiligen Landes zugeschnittene Strategien entwickelt, auf deren Grundlage auch der Einsatz negativer Instrumente die Anerkennung des Drittstaates finden soll. ${ }^{23}$

\section{,Demokratisierungs-Stabilitätsdilemma ‘}

Beim oben aufgezeigten Zusammenhang zwischen Demokratie und Stabilität muss mitbedacht werden, dass Demokratisierungsprozesse in bestimmten Ländern - je nach Resonanzstruktur - destabilisierend wirken können, woraus sich für die Europäische Union ein Zielkonflikt ergibt. Im Hinblick auf die Mittelmeerpolitik wird dieser Zielkonflikt von Annette Jünemann als ,Demokratisierungs-Stabilitätsdilemma ' bezeichnet. ${ }^{24}$ Demzufolge ist es hoch wahrscheinlich, dass demokratische Reformen in den autoritären Systemen der arabischen Mittelmeerländern ${ }^{25}$ turbulente Transformationsprozesse hervorbringen und damit zunächst einmal destabilisierend wirken würden. Mögliche Szenarien sind der Ausbruch von Bürgerkriegen, der Zerfall von Staaten und/oder die (eventuell sogar demokratische) Machtübernahme antiwestlicher Islamisten. Angesichts dieser Risiken ist es wenig verwunderlich, dass die Europäische Union im Zielkonflikt zwischen Demokratisierung und Stabilisierung letzterer den Vorzug gibt. Obwohl die Mittelmeerdrittländer Partner der ,Neuen Nachbarschaftspolitik' sind, wird hier das für alle geltende demokratiepolitische Instrumentarium bewusst nur halbherzig implementiert; die Gemeinschaft setzt im südlichen Mittelmeerraum aus stabilitätspolitischen Gründen auf die bewährte Kooperation mit autoritären Systemen. Opfer dieser Strategie sind vor allem die Vertreter der demokratischen Zivilgesellschaften im südlichen Mittelmeerraum, denn der, low-profile‘ Strategie entsprechend bevorzugt die Kommission bei der Suche nach adäquaten Projektpartnern nun wieder Organisationen, die sich bereits in legalen staatlichen Strukturen bewegen. Dieses Vorgehen, das hinter die be-

21 Der Theorie des demokratischen Frieden ist relativierend entgegenzuhalten, dass Demokratien nur untereinander aber nicht prinzipiell friedlicher sind als undemokratische Regime.

22 Vgl. Marise Cremona: The European Neighbourhood Policy: Legal and Institutional Issues, Workshop Promoting Democracy and the Rule of Law: American and European Strategies and Instruments, 4-5.10.2004, Standford University. Vgl. hierzu auch die EU-Sicherheitsstrategie von 2003, abrufbar unter: http://www.consi lium.europa.eu/uedocs/cmsUpload/031208ESSIIDE.pdf (letzter Zugriff: 15.08.2006).

23 Vgl. Marise Cremona: The European Neighbourhood Policy, S. 7. Zur Nachbarschaftspolitik vgl. auch Elena Baracani: Pre-accession and Neighbourhood: The European Union's Democratic Conditionality in Turkey and Morocco. In: Annette Jünemann/Michèle Knodt (Hrsg.): The EU as an External Democracy Promoter.

24 Vgl. Annette Jünemann: Security Building in the Mediterranean.

25 Die politischen Systeme der einzelnen Mittelmeerdrittländer unterscheiden sich zwar stark voneinander, haben in Bezug auf ihre demokratischen Standards jedoch Gemeinsamkeiten, die die verallgemeinernde Bezeichnung autoritär trotz aller Differenzen rechtfertigen. 
reits erwähnten Lernprozesse zurückfällt, wird vor allem vom Europäischen Parlament als Kooperation mit der falschen Zielgruppe scharf kritisiert. ${ }^{26}$

Dass sich die Europäische Union im Zielkonflikt zwischen Demokratisierung und Stabilisierung im Zweifelsfall für die Stabilisierung entscheidet, bestätigt eine Reihe von Studien zu Staaten ohne Beitrittsperspektive: Genannt seien hier die Fallbeispiele Äthiopien, Südafrika (vor dem Ende der Apartheid), Zentralasien und Jemen. ${ }^{27}$ Aus konstruktivistischer Perspektive kann zusammenfassend festgestellt werden, dass bei der Lösung des Demokratisierungs-Stabilitätsdilemmas die außenpolitische Rolle der Europäischen Union als ,internationaler Sicherheitsagent" offensichtlich über ihre außenpolitische Rolle als ,internationaler Demokratieförderer' dominiert, es sei denn, es handelt sich um ein Land mit Beitrittsperspektive. Mitgliedstaaten, die durch die potenzielle Destabilisierung von Drittstaaten direkt betroffen wären, unterstützten diese Rollenhierarchie - in Einklang mit ihren jeweiligen außenpolitischen Rollen als „Wahrer der nationalen Interessen.“28

\section{Das veränderte Sicherheitsparadigma}

Stabilitätsüberlegungen werden auch durch Veränderungen in der internationalen Umwelt der Gemeinschaft beeinflusst. Eines der eindrücklichsten Beispiele für diesen Zusammenhang ist die internationale Sicherheitssituation nach dem 11. September 2001. In dem von Annette Jünemann herausgegebenen Sammelband ,Euro-Mediterranean Relations after September 11“29 analysieren Spezialisten der Region die Entwicklung der EU-Politik im Mittelmeerraum vor dem Hintergrund dieses einzigartigen Paradigmenwechsels in der internationalen Umwelt. Übereinstimmend kommen die Autorinnen und Autoren zu dem Ergebnis, dass ,9/11' eine weitere Verschiebung zugunsten des Sicherheitsparadigmas im Umgang mit allen ,islamischen' Ländern verursachte. Infolgedessen reduzierte die Europäische Union ihre Reformbemühungen in der Region so weit gehend, dass man von einer faktischen Einstellung der Demokratieförderung sprechen kann - zugunsten der Stabilisierung der autoritären Regime dieser als Sicherheitsrisiko wahrgenommenen Staaten. Damit wurde die Demokratieförderung als langfristige und auf Nachhaltigkeit angelegte Strategie der Sicherheits- und Stabilisierungspolitik aufgegeben. Ähnliche Auswirkungen von ,9/11` auf die EU-Demokratieförderung wurden in Äthiopien und Zentralasien verzeichnet. ${ }^{30}$

26 Vgl. Interview von Annette Jünemann mit Mitgliedern des Europäischen Parlaments am 25. April 2006 in Brüssel. Hierbei spielt auch eine Rolle, dass die Kommission ihre Arbeit dezentralisiert hat. Die Identifikation geeigneter Projektpartner wird zunehmend von den EU-Delegationen vor Ort wahrgenommen. Dieses Verfahren hat sich insofern als ungeeignet erwiesen, als die EU-Delegationen sich zur diplomatischen Rücksichtnahme auf die Regierungen im jeweiligen Gastland verpflichtet sehen.

27 Vgl. Stefan Brüne: Testfall Äthiopien: Die neue Afrikastrategie der Europäischen Union; Siegmar Schmidt: EU Democracy Assistance in Africa; Alexander Warkotsch: Die Demokratisierungspolitik der EU in Zentralasien; Richard Youngs: Democracy Dilemmas in the Middle East. The Cases of Iran, Saudi Arabia and Yemen. Jeweils in: Annette Jünemann/Michèle Knodt (Hrsg.): The EU as an External Democracy Promoter.

28 Die Rollentheorie geht von der Annahme aus, dass internationale Akteure bestimmte Rollen übernehmen, die fortan ihre außenpolitischen Interessen und Handlungen beeinflussen. Das außenpolitische Rollenprofil eines internationalen Akteurs wird jedoch nicht nur von den internen Diskursen - hier den Diskursen innerhalb des EU-Mehrebenensystems - beeinflusst, sondern auch von Veränderungen im internationalen Kontext und nicht zuletzt von den sich wandelnden Rollenerwartungen externer Akteure. Insofern ist es durchaus möglich, dass die derzeitige Prioritätensetzung zwischen den konfligierenden Rollenkonzepten der EU sich wieder umkehrt, und zwar zugunsten der Rolle des internationalen Förderers der Demokratie. Vgl. Annette Jünemann: Realpolitisches Nutzenkalkül oder konstruktivistischer Rollenkonflikt? Erklärungsansätze für die Inkohärenz europäischer Demokratieförderung im südlichen Mittelmeerraum, in: Annette Jünemann/Michèle Knodt (Hrsg.): The EU as an External Democracy Promoter.

29 Vgl. Annette Jünemann (Hrsg.): Euro-Mediterranean Relations.

30 Vgl. Stefan Brüne: Testfall Äthiopien; Alexander Warkotsch: Die Demokratisierungspolitik der EU in Zentralasien. Jeweils in: Annette Jünemann/Michèle Knodt (Hrsg.): The EU as an External Democracy Promoter. 
Dieser Entwicklung stehen allerdings Tendenzen innerhalb der Gemeinschaft gegenüber, sich im Internationalen System, insbesondere gegenüber den USA, verstärkt als ,normative power" zu profilieren. ${ }^{31}$ Dieses Profilierungsbemühen ist zunächst einmal eine Erklärung für die offensichtliche Diskrepanz zwischen der starken demokratiepolitischen Rhetorik einerseits und ihrer defizitären praktischen Umsetzung andererseits. Möglich ist aber auch, dass mit der Profilierung als ,normative power' erste Ansätze eines grundsätzlichen Prioritätenwechsels in der EU-Außenpolitik sichtbar werden, zugunsten einer konsequenteren und kohärenteren externen EU-Demokratieförderung.

\section{Fazit und Anschlussmöglichkeiten}

Die hier skizzierten Ergebnisse unseres Forschungsprojektes zur externen Demokratieförderung der Europäischen Union haben vor allem eins gezeigt: Der Bedarf an systematisch vergleichender Forschung im Feld ist groß. Als weitgehend abgeschlossen kann man die Erforschung der Demokratieförderung im Kontext der EU-Erweiterung bezeichnen. Dies betrifft in erster Linie alle Länder, die der EU im Mai 2004 nach einem langjährigen Anpassungsprozess beigetreten sind. In den Ländern, die jetzt noch über eine Beitrittsperspektive verfügen, wie die Balkanländer, oder die sich mit einiger Berechtigung eine Beitrittsperspektive erhoffen, wie die Ukraine, bemüht sich die Gemeinschaft um eine konsequente Demokratisierungspolitik. Alle verfügbaren Instrumente werden auch implementiert.

Angesichts der Probleme, die die Bewältigung der letzen Beitrittswelle der Europäischen Union bereitet, ist davon auszugehen, dass der Beitrittsprozess mit der Aufnahme der Balkanländer und eventuell noch der Ukraine zu einem zumindest vorläufigen Ende kommen wird. Damit entsteht neuer Forschungsbedarf, denn es hat sich gezeigt, dass die EU-Demokratieförderung in Drittländern ohne Beitrittsperspektive von einer Vielzahl, zum Teil interdependenter, Faktoren abhängig ist. Weitergehender Forschung bleibt es vorbehalten, die Erklärungskraft der einzelnen Faktoren beziehungsweise deren Interaktionseffekte zu ergründen.

Untrennbar von der hier bearbeiteten Frage nach der Strategie- und Instrumentenwahl stellt sich die Frage nach deren Wirkung in unterschiedlichen Drittstaaten. Ausgehend von der Annahme, dass die unterschiedliche Wirkung der EU-Demokratieförderung besonders von der innenpolitischen Konstellation eines betroffenen Landes abhängt, sind vor allem solche Staaten von besonderem Interesse, die fragil sind und über kaum gefestigte staatliche Strukturen verfügen. Um diesen Fällen gerecht zu werden, muss sowohl die Wissenschaft als auch die Praxis sich von ihrer bisherigen Staatszentrierung lösen und neue Wege suchen. ${ }^{32}$

31 Dies wurde zuletzt im Kontext der Bemühungen der USA um eine transatlantische Greater-Middle-East Initiative deutlich: Nachdem der amerikanische Vorstoß zu deutlich erkennen ließ, dass es sich um ein Konzept westlicher Hegemonialpolitik handelte, das lediglich unter dem positiv besetzten Begriff der Demokratisierungspolitik firmierte, verweigerte die Europäische Union ihre Kooperation und setzte sich für ein neues Konzept ein, dass europäischen Vorstellungen von externer Demokratieförderung näher kam und unter dem Titel Broader Middle East Initiative bekannt wurde. Vgl. U.S. Department of State: Partnership for Progress and a Common Future with the Region of the Broader Middle East and North Africa. Fact Sheet, The White House Office of the Press Secretary, 9. Juni 2004. Zitiert in: Annette Jünemann: Demokratie für die Arabische Welt: Anmerkungen zu einem sperrigen Exportartikel, in: Hans-Georg Ehrhart/ Margret Johannsen (Hrsg.): Herausforderung Mittelost: Übernimmt sich der Westen?, Baden-Baden 2005, S. 115-127.

32 Diese Skizze der noch unbeantworteten Fragen verdeutlicht, dass die Erforschung der externen Demokratieförderung der Europäischen Union eine große Baustelle ist, auf der viele Kolleginnen und Kollegen gleichzeitig arbeiten. Zur Vernetzung der unterschiedlichen Forschungsprojekte soll eine von uns eingerichtete Webseite (http://www.ifs.tu-darmstadt.de/democracy) dienen. 